\title{
Die Bindungsweise des Schwefels im Eiweis.
}

von

Fr. N. Schnlz.

(Aus dem physiologisch-chemischen Institut zu Strassburg. Neue blge Nr. 6.) (Der Redaction zugegangen am 17. Februar 1898.)

\section{Einleitung.}

Für die Beantwortung der Frage nach der Azahl der Schwefelatome, die in einem Eiweissmoleküle vorhanen sind, ist es von Wichtigkeit, zu wissen, ob der Schwefel tch darin in einer oder in verschiedenen Formen vorfindet, un' welches gegebenen Falls das Verhältniss der verschiedenen Frmen zu einander ist. Mulder, ${ }^{1}$ ) der der Erste war, der die ledeutung des Schwefels im Eiweissmolekül gebührend hervorhol glaubte, dass sich aller Schwefel durch Kochen mit Alkali als ichwefelmetall abspalten lasse, eine Annahme, auf die sich am Theil seine vielumstrittene Proteintheorie aufbaute. Im Kamfe grgen diese Proteintheorie führte Fleitmann, ${ }^{2}$ ) ein Schüler liebig's, als Erster den Nachweis, dass man durch noch 9 langes Kochen mit Alkali nur einen Bruchtheil des Gesammtchwefels als Schwefelmetall abspalten kann, während ein sehr eträchtlicher Theil zurückbleibt, welcher sich auf anderen Wege,

1) S. die Zusammenstellung in Gmelin-Kraut's Hadbuch d. org. Chemie. 7. Bd., 1870, S. 2198-2208.

2) Liebig's Annalen. Bd. 61, 1847, S. 121-125 und Bd66, 1848,. S. $380-381$. 
z. B. durch Schmelzen mit Kali und Salpeter, nachweisen lässt. Er nahm deshalb zwei verschiedene Formen von Schwefel im Eiweissmolekül an und bestimmte das Mengenverhältniss derselben zu einander. Die späteren Untersucher auf diesem Gebiete, Nasse, ${ }^{1}$ ) Danilevsky, ${ }^{2}$ ) Krüger, ${ }^{3}$ ) Suter, ${ }^{4}$ ) Malerba, $\left.{ }^{5}\right)$ haben die alten Angaben Fleitmann's im Wesentlichen bestätigt und durch neue Angaben über das Mengenverhältniss der beiden Schwefelformen erweitert, wobei sie aber durchaus nicht zu übereinstimmenden Zahlenergebnissen gelangten.

Die eigentliche Abspaltung des Schwefels wurde in allen Versuchen durch Kochen mit Kali- oder Natronlauge von wechselnder Concentration in Gegenwart von Blei- oder Wismutoxyd vorgenommen, sei es wie bei $\mathrm{Kr}$ ïg er in offener Porzellanschaale, sei es in der Kochflasche am Kühler oder Steigrohr. Die Bestimmung der nach dem Kochen vorhandenen Menge von Schwefelmetall wurde von den verschiedenen Untersuchern in verschiedener Weise ausgeführt. Fleitmann (und ebenso Danilevsky) übersäuerte nach dem Kochen mit Essigsäure, filtrirte, wusch aus, schmolz Filter und Rückstand im Silbertiegel mit Kali und Salpeter und bestimmte die hierbei gebildete Schwefelsäure als schwefelsauren Baryt. Krüger oxydirte das Schwefelmetall mit Chlorwasser, führte das gebildete schwefelsaure Blei durch Zusatz von Ammoniumcarbonat in schwefelsaures Ammonium und Bleicarbonat über. Von letzterem wurde abfiltrirt, im Filtrat wurde das überschüssige Ammoniumcarbonat zunächst verjagt, sodann die Schwefelsäure mit Baryumchlorid ausgefällt. Malerba wandte ein indirektes Verfahren an, indem er nicht den Schwefelgehalt des Schwefelbleiniederschlags, sondern den des Filtrates durch Schmelzen mit Soda und Salpeter unter Anwendung der von Hammarsten ${ }^{\theta}$ ) hervorgehobenen Vorsichtsmassregeln bestimmte. Suter wog den auf dem Filter gesammelten und getrockneten Niederschlag und berechnete, indem er denselben für Schwefelblei ansah, hieraus den Schwefelgehalt. Die von den einzelnen Untersuchern gefundenen Werthe sind in der folgenden Zusammenstellung aufgeführt, in welcher unter a der Gesammtschwefel, unter b der abspaltbare Schwefel in Procenten ersichtlich gemacht ist.

1) Studien über die Eiweisskörpær III. Pflüger's Arch. Bd. VIII,1874.

2) Zeitschr. f. physiol. Chem. Bd. VII, 1883, S. 427-449.

3) Pflüger's Archiv. Bd. 43. 1888.

4) Zeitschr. f. physiol. Chem. Bd. XX, 1895, S. 564-582.

5) Rendic. della R. Accad. delle scienze di Napoli fasc. 3-5, 1894.

6) Zeitschr. f. physiol. Chem. Bd. IX, S. 273. 


\begin{tabular}{|c|c|c|c|c|c|c|c|c|c|c|c|}
\hline $\begin{array}{l}\text { Untersuchter } \\
\text { Eiweisskörper }\end{array}$ & Fleitr & $\begin{array}{c}\text { nann } \\
b\end{array}$ & $\begin{array}{c}\text { Danil } \\
\mathbf{a}\end{array}$ & $\begin{array}{c}\text { vsky } \\
\text { b }\end{array}$ & $\begin{array}{l}\text { Krü } \\
\mathrm{a} \quad 1\end{array}$ & & $\mathrm{Su}$ & & $\begin{array}{l}\text { Mal } \\
\mathrm{a}\end{array}$ & lerba & $\begin{array}{c}\text { Gürber } \\
a: b\end{array}$ \\
\hline Serun & • & $\begin{array}{l}0,76 \\
1,19 \\
1,03\end{array}$ & • & • & - & • & & • & 1,75 & 0,41 & $\left.5: 3^{3}\right)$ \\
\hline Eieralbumin & . & . & 2,0 & 1,2 & 1,66 & 0,44 & . & . & 1,71 & 0,49 & . \\
\hline Fibrin ........ & . & 0,52 & 1,2 & 0,4 & 1,20 & 0,38 & . & . & 1,14 & 0,41 & . \\
\hline Casein ........ & • & 0,07 & 1,2 & 0,4 & - & - & $\cdot$ & . & . & $\bullet$ & - \\
\hline «Pepton»(Witte) & . & . & • & - & . & . & 0,65 & 0,34 & 1,65 & 0,47 & - \\
\hline Keratin . & • & $\bullet$ & - & - & - & . & $\left(\begin{array}{l}2,521) \\
2,662\end{array}\right.$ & $\begin{array}{l}2,34 \\
1,29\end{array}$ & 4,43 & 0,80 & - \\
\hline $\begin{array}{l}\text { Eihäutchen ..... } \\
\text { Glutin ........ }\end{array}$ & $\begin{array}{c}4,15 \\
.\end{array}$ & $\begin{array}{c}2,51 \\
\cdot\end{array}$ & . & • & . & - & • & · & $\dot{1,15}$ & 0,44 & . \\
\hline
\end{tabular}

Die grossen Abweichungen in den Angaben der einzelnen Untersucher sind erklärlich, wenn man bedenkt, dass nicht von sicher reinem und einheitlichem Material ausgegangen wurde, und dass der Untersuchungsweise später zu erwähnende Fehler anhaften, deren Tragweite nicht ohne Weiteres abzusehen ist.

Es erschien daher zweckmässig nach einer Methode, deren Zuverlässigkeit an bekannten Körpern quantitativ erprobt war, diese Bestimmungen an reinem und einheitlichem Material $z u$ wiederholen.

\section{Quantitative Versuche über die Abspaltbarkeit des Schwefels.}

\section{A. Methodik.}

Die wichtigste Klippe für die quantitative Bestimmung des durch Kochen mit Alkali und Schwefelmetall abspaltbaren Schwefels stellt die nachträgliche Oxydation durch den Sauerstoff der Luft dar. Den Beweis für das Stattfinden einer

1) Das Untersuchungsobject waren Haare.

2) Das Untersuchungsobject waren Federn.

3) Gürber gibt die angeführten, offenbar von eigenen Untersuchungen herrührenden Zahlen für krystallisirendes Serumalbumin in dem jüngst erschienenen Leitfaden der Physiologie von Schenk und Gürber. 
solchen Oxydation hat schon Fleitmann beigebracht, indem er zeigte, dass Eiweiss, mit Kalilauge in offener Schaale gekocht, nur während der ersten Stunden auf Zusatz von Blei eine Bildung von Schwefelblei zeigt, während von da ab keine Schwefelreaction durch Blei mehr zu erkennen ist. "Die Oxydation durch den Sauerstoff der Atmosphäre hielt also gleichen Schritt mit der Entziehung des Schwefels durch das Kali.» Setzt man beim Kochen einer Eiweisslösung mit Alkali Bleioder Wismutsalz hinzu, so tritt der abgespaltene Schwefelwasserstoff an das Metall, wodurch die oxydirende Wirkung des Sauerstoffs der Luft weniger auffällig wird, wie in dem von Fleitmann beobachteten Fall, da Schwefelblei und Schwefelwismut sich weniger leicht oxydiren, wie Schwefelalkali. Immerhin können, wie quantitative Versuche mit Schwefelblei mich lehrten, auch davon recht beträchtliche Mengen der Oxydation anheim fallen.

Um diese Gefahr auszuschliessen, wurde in der Mehrzahl der Versuche beim Kochen feingeraspeltes Zink ${ }^{1}$ ) als Reductionsmittel zugesetzt. In einem anderen Theil der Versuche wurde das Kochen ohne Zusatz von Zink in Leuchtgasatmosphäre vorgenommen, und zwar wurde hierbei das seitlich der auf einem Sandbad stehenden Kochflasche zugeführte Leuchtgas, nachdem es diese und den Kühler durchstrichen, zum Heizen des Sandbades benutzt.

Die Bestimmung des nach dem Kochen als Schwefelmetall vorhandenen Schwefels wurde anlehnend an das Verfahren von Fleitmann in folgender Weise ausgeführt. Es wurde mit Essigsäure übersäuert, filtrirt und ausgewaschen. Filter und Rückstand wurden mit Soda und Salpeter (im Verhältniss 3:2) geschmolzen, die Schmelze in Wasser gelöst und die Lösung nach Durchleiten von Kohlensäure filtrirt. Das Filtrat wurde zum Verjagen der Salpetersäure mehrmals mit starker Salzsäure auf dem Wasserbad zur Trockne verdampft. Der salpetersäurefreie Rückstand, in Wasser gelöst, wurde mit Baryumchlorid ausgefällt und die gebildete Menge Baryumsulfat durch Wägung bestimmt.

1) Die gewöhnlichen, käuflichen Zinksorten sind schwefelhaltig, also zu den vorliegenden Versuchen nur unter Anwendung einer Correctur zu verwenden. Ich benutzte von Merck und von Schuchardt bezogene Zinkpräparate, die völlig schwefelfrei waren. 
Dies Verfahren liefert ausreichend genaue Werthe, wie Kontrollversuche mit bekannten Mengen Schwefelblei zeigten.

0,355 gr. reines Schwefelblei 1 ) lieferten 0,346 gr. Baryumsulfat.

Verlangt waren $0,346 \mathrm{gr}$.

0,4497 gr. Schwefelblei lieferten 0,4415 gr. Baryumsulfat.

Verlangt waren $0,439 \mathrm{gr}$. Baryumsulfat.

Die Versuche nach der Zinkmethode wurden in der Weise ausgeführt, dass die zu untersuchende Substanz mit ca. 1 gr. fein geraspeltem Zink und ungefähr $50 \mathrm{ccm} .30 \%$ iger Natronlauge, der einige Tropfen einer concentrirten Bleiacetatlösung oder frisch gefälltes Wismutoxyd zugesetzt waren, am Rückflusskühler oder Steigrohr auf dem Sandbade gekocht wurde. $\left.{ }^{2}\right)$ Hierbei übt das Zink eine reducirende Wirkung aus, die den Einfluss des atmosphärischen Sauerstoffs paralysirt, aber sich unter anderem auch darin äussert, dass das in Lösung befindliche Bleioxyd als metallisches Blei niedergeschlagen wird, so dass der Lösung namentlich beim Kochen in kurzer Zeit alles Bleioxyd entzogen wird. Dies hat den Nachtheil, dass das abgespaltene Schwefelalkali nur schwer auf das metallische Blei reagirt. Diese reducirende Wirkung des Zinks lässt allmählich nach, besteht aber auch nach vielstündigem Kochen fort. Es ist deshalb zweckmässig, von Zeit zu Zeit, anfangs häufiger,

1) Reines Schwefelblei stellt man zweckmässig dar durch Ausfällen einer Bleiacetatlösung mit Schwefelwasserstoff und Glühen des ausgewaschenen Niederschlags im Schwefelwasserstoff-, sodann im Wasserstoffstrom.

0,739 gr. eines so dargestellten Präparates, im Tiegel mehrmals mit Salpetersäure und dann mit Schwefelsäure abgeraucht, lieferten 0,931 gr. schwefelsaures Blei (verlangt $0,937 \mathrm{gr}$.). 0,600 gr.).

$0,473 \mathrm{gr}$. ebenso behandelt lieferten $0,597 \mathrm{gr}$. Bleisulfat (verlangt

2) Zur Verbindung der Kochflasche mit dem Kühler darf man keine gewöhnlichen Gummistopfen verwenden, die mit Schwefel imprägnirt sind und hiedurch einen Analysenfehler verursachen können. Zweckmässig versieht man einen Korkstopfen mit der entsprechenden Bohrung, schneidet denselben dann der Länge nach in 2 gleiche Hälften, die man getrennt von einander mit mehrfacher Lage von Stanniolpapier überzieht. Dann befestigt man die beiden Hälften mit einer Drahtschlinge an dem Kühler und kann nun durch Eindrücken in den Flaschenhals einen völlig genügenden Verschluss erzielen, der beim Kochen nicht angegriffen wird. 
später alle ein bis zwei Stunden, etwas neues Bleiacetat hinzuzufügen.

\section{B. Versuche.}

In dieser Weise wurden zunächst schwefelsaures Natrium, schwefligsaures Natrium, methylschwefelsaures Kali und Taurin untersucht. Auch nach 10 stündigem Kochen schied sich hierbei kein Schwefelblei ab.

Die reducirende Wirkung des Zinks genügt also nicht, um aus diesen Verbindungen, deren Schwefel in direkter Verbindung mit Sauerstoff steht, Schwefelmetall zu bilden.

Ein sehr lehrreiches Beispiel bietet das unterschwefligsaure Natrium, das Natriumthiosulfat.

Kocht man unterschwefligsaures Natrium an der Luft mit $30 \%$ iger Natronlauge, der etwas Bleiacetat zugesetzt ist, so tritt keine Spur von Schwärzung auf, auch nicht nach stundenlangem Kochen. ${ }^{1}$ ) Setzt man dagegen $\mathrm{zu}$ einer Lösung von Thiosulfat in $30 \%$ iger Natronlauge etwas geraspeltes Zink, so tritt bald eine reichliche Abspaltung von Schwefel ein, die sich durch intensive Schwärzung bei Zusatz von Blei äussert. Quantitative Untersuchungen ergaben, dass, wenn man in der richtigen Weise verfährt, schliesslich genau die eine Hälfte des im Thiosulfat vorhandenen Schwefels in Form von Schwefelmetall abgespalten wird. Bei zahlreichen Versuchen habe ich nie mehr wie die Hälfte des Gesammtschwefels erhalten können, dagegen oft genug bei fehlerhafter Versuchsanordnung etwas weniger. Die maximalen Werthe erhält man, wenn man in der vorher beschriebenen Weise verfährt und das Kochen einige Stunden fortsetzt.

Die Abspaltung des Sichwefels erfolgt zuerst sehr rasch, so dass in der ersten halben Stunde ca. 75\% des überhaupt abspaltbaren Schwefels als Sulfid erhalten werden, zum Schluss nur sehr langsam. Der Sicherheit halber lässt man zweck-

1) Lässt man eine Lösung von Thiosulfat in $30 \%$ iger Natronlauge, der etwas Blei hinzugesetzt ist, auf dem Sandbade eindicken, so tritt bei hoher Concentration an Alkali eine allmählich zunehmende Bildung von Schwefelblei ein. (S. auch die Leuchtgasversuche auf Seite 34.) 
mässig das Kochen etwa 10 Stunden dauern. Als Beispiele mögen folgende Analysen dienen:

1. 0,3082 gr. $\mathrm{Na}_{2} \mathrm{~S}_{2} \mathrm{O}_{3}$ (Bleizusatz) ergaben 0,283 gr. $\mathrm{BaSO}_{4}$. Die $0,3082$ gr. enthielten insgesammt $0,0793 \mathrm{gr} . \mathrm{S} 1)=0,0578 \mathrm{gr}$. $\mathrm{BaSO}_{4}$; es wurden also 48,9\% des Gesammtschwefels gefunden.

2. 0,2539 gr. $\mathrm{Na}_{2} \mathrm{~S}_{2} \mathrm{O}_{3}$ (Bleizusatz) ergaben 0,2314 gr. $\mathrm{BaSO}_{4}$. Verlangt waren $0,476 \mathrm{gr} . \mathrm{Ba} \mathrm{SO}_{4}$; also gefunden $48,6 \%$

3. 0,266 gr. $\mathrm{Na}_{2} \mathrm{~S}_{2} \mathrm{O}_{3}$ (Wismutzusatz2) ergaben 0,2444 gr. $\mathrm{BaSO}_{4}$. Verlangt waren $0,498 \mathrm{gr} . \mathrm{BaSO}_{4}$; also gefunden $49,1 \%$

4. 0,304 gr. $\mathrm{Na}_{2} \mathrm{~S}_{2} \mathrm{O}_{3}$ (Wismutzusatz) ergaben 0,2823 gr. $\mathrm{Ba} \mathrm{SO}_{4}$. Verlangt waren $0,570 \mathrm{gr}$. $\mathrm{BaSO}_{4}$; also gefunden $49,5 \%$.

Da im unterschwefligsauren Natrium die eine Hälfte des Schwefels nicht in direkter Verbindung mit Sauerstoff steht, und diese eine Hälfte sich nach der vorbeschriebenen Methode quantitativ abspalten lässt, ist es wahrscheinlich gemacht, dass diese Methode sich allgemein zum quantitativen Nachweis des als Schwefelmetall abspaltbaren Schwefels eignet.

Zur Prüfung der Methode wurde weiter Sulfoharnstoff untersucht. Wurde Sulfoharnstoff 2 Stunden mit alkalischer Bleilösung gekocht ohne Zusatz von Zink und dann der entstandene Niederschlag nach dem modificirten Fleitmann'chen Verfahren auf seinen Schwefelgehalt untersucht, so wurden $94-96 \%$ des verlangten Schwefels gefunden. Der Verlust durch Oxydation

1) Thiosulfat krystallisirt mit 5 Molekülen Krystallwasser; da es an feuchter Luft etwas Wasser anziehen kann und die Krystalle beim Trocknen im Exsiccator verwittern, wurde das Analysenmaterial in der Weise gewonnen, dass krystallisirtes Thiosulfat zerkleinert, im Exsiccator bis zum beginnenden Verwittern getrocknet, nunmehr nochmals fein pulverisirt und in einem gut schliessenden Wägegläschen an trockner Stelle (nicht im Exsiccator) aufbewahrt wurde. (Das Wägegläschen behielt constantes Gewicht.) In dem so erhaltenen Pulver wurde der Gesammtschwefel durch Schmelzen mit Natriumsuperoxyd und Soda (nach v. A sboth) bestimmt.
1) 0,1278
gr. $\mathrm{Na}_{2} \mathrm{~S}_{2} \mathrm{O}_{3}$ lieferten 0,2389 gr. $\mathrm{Ba} \mathrm{SO}_{4}$ $=0,0328 \mathrm{gr} . \mathrm{S}=25,66 \%$
2) 0,1175
gr. $\mathrm{Na}_{2} \mathrm{~S}_{2} \mathrm{O}_{3}$ lieferten 0,2204 gr. $\mathrm{BaSO}_{4}$ $=0,0303$ gr. $\mathrm{S}=25,79 \%$
Mittel $25,72 \%$

2) Die mit Wismut ausgeführten Analysen ergaben etwas höhere Werthe, es wurde daher im Nachfolgenden zu den Analysen meist Wismut verwandt. 
ist in diesem Fall nicht bedeutend, da 2stündiges Kochen zur völligen Abspaltung des Schwefels genügt.

Nach dem oben beschriebenen Verfahren unter Zusatz von Zink ergaben sich folgende Resultate:
1) 0,0341 gr. Sulfoharnstoff $=0,0143$ gr. $S$
lieferten 0,1025 gr. $\mathrm{Ba} \mathrm{SO}_{3}=0,0141$ gr. S;
also gefunden $98,6 \%$.
2) 0,0601 gr. Sulfoharnstoff $=0,0253$ gr. $\mathrm{S}$
lieferten $0,184 \mathrm{gr} . \mathrm{Ba} \mathrm{SO}_{4}=0,252 \mathrm{gr}$. S; also gefunden $99,6 \%$.

Zur weiteren Sicherstellung der Methode wurde Thioessigsäure untersucht. ${ }^{1}$ ) Das nicht weiter getrocknete Präparat ergab in einem Versuche $28,2 \%$, in einem anderen $27,4 \%$. abspaltbaren Schwefel.

In beiden Fällen wurde das von dem Schwefelbleiniederschlag erhaltene Filtrat eingedampft, mit Soda und Salpeter im Nickeltiegel geschmolzen und dann auf Schwefel geprüft. Die Probe fiel völlig negativ aus, ein Beweis dafür, dass aller Schwefel abgespalten war und dass keine 0xydation des abgespaltenen Schwefels während des Kochens stattgefunden hatte.

Es wurden weiter Versuche mit Cystin angestellt. Verwandt wurde ein aus einem Cystinstein stammendes Präparat, welches genau den verlangten Schwefelgehalt aufwies.

Gesammtschwefel (nach v. Asboth bestimmt): 0,0618 gr. lieferten 0,1194 gr. $\mathrm{Ba} \mathrm{O}_{4}$; verlangt waren 0,120 gr. $\mathrm{Ba} \mathrm{S}_{4}$.

Das Ergebniss der Abspaltungsversuche ist aus der folgenden Zusammenstellung ersichtlich.

Versuch I. 0,128 gr. Cystin wurden mit $1 \mathrm{gr} . \mathrm{Zn}, 50 \mathrm{ccm}$. Natronlauge und üherschüssigem Wismutoxyd 10 Stunden gekocht. Gefunden. wurden $0,1327 \mathrm{gr}$. $\mathrm{Ba} \mathrm{S} \mathrm{O}_{4}$; verlangt waren $0,248 \mathrm{gr}$., also gefunden. $5: 3 \%$ des Gesammtschwefels.

Versuch II. 0,119 gr. Cystin, wie in Versuch I. behandelt und 25 Stunden gekocht. Verlangt $0,231 \mathrm{gr}$. $\mathrm{BaS}_{4}$, gefunden 0,124 gr. = $53,7 \%$.

Versuch III. 0,0כ0 gr. Cystin mit 1 gr. Zn, überschüssigem Wismut und ca. $30 \mathrm{ccm}$. Natronlauge in einer Einschmelzröhre in Wasserstoffatmosphäre 10 Stunden auf $105-110^{\circ}$ erhitzt. Verlangt $0,0968 \mathrm{gr}$. $\mathrm{BaS}_{4}$, gefunden 0,0507 gr. $\mathrm{Ba} \mathrm{S}_{4}=52,4 \%$.

1) Verwandt wurde ein von Kahlbaum bezogenes Präparat. 
Versuch IV. 0,1067 gr. Cystin wurden mit $30 \%$ Natronlauge (mit Blei) 10 Stunden in Leuchtgasatmosphäre gekocht. Gefunden $0,0628 \mathrm{gr}$. $\mathrm{Ba} \mathrm{S} \mathrm{O}_{4}$, verlangt 0,204 gr. $\mathrm{Ba} \mathrm{S} \mathrm{O}_{4}$, also gefunden $30,7 \%$.

In Versuch I und II liess sich nach Abfiltriren des Schwefelwismutniederschlages im mit Essigsäure versetzten Filtrat Schwefelsäure nachweisen; in Versuch III und IV fiel die Probe naturgemäss negativ aus. Wurden die mit Essigsäure sauer gemachten Filtrate auf dem Wasserbade eingeengt, so bildete sich ein reichlicher schwarzer Niederschlag, der auf Zusatz von Salzsäure Schwefelwasserstoff entwickelte. (Bei Versuch IV war dieser Niederschag besonders reichlich.)

Bei Versuch III wurde in der Hälfte des essigsauren Filtrates die beim Einengen gebildete Menge Schwefelwismut bestimmt, und zwar wurden $0,0148 \mathrm{gr} . \mathrm{Ba} \mathrm{S} \mathrm{O}_{4}$ gefunden, somit im ganzen Filtrat noch $30,6 \%$ des Gesammtschwefels des verwandten Cystins; neben dem Schwefelwismut war nunmehr auch Schwefelsäure nachweishar. Die zweite Hälfte des essigsauren Filtrates von Versuch III, die, wie erwähnt, keine Schwefelsäure enthielt, wurde mit grossem Ueberschuss von reinem Natriumhydroxyd von Neuem 7 Stunden gekocht. Hierbei bildete sich kein Schwefelwismut, dagegen war nunmehr reichlich Schwefelsäure nachweisbar.

Wurde Cystin mit Eisessig, dem etwas Bleiacetat zugesetzt war, gekocht, so trat keine Abspaltung von Schwefelwạsserstoff ein.

Gystein (nach der Baumann'schen Methode durch Reduction aus Cystin dargestellt) verhielt sich gegenüber Eisessig ebenso. Auch damit wurde ein Abspaltungsversuch nach dem vorbeschriebenen Verfahren gemacht.

0,052 gr. Cystein (mit Wismut) 10 Stunden gekocht, lieferten $0,052 \mathrm{gr} . \mathrm{Ba} \mathrm{S} \mathrm{O}_{4}$ statt $0,100 \mathrm{gr}$., die verlangt waren; also $52 \%_{0}$.

Auch hier enthielt das essigsaure Filtrat reichlich Schwefelsäure und bildete beim Einengen Schwefelwismut.

Die Versuche mit Cystin bezw. Cystein haben also gezeigt, dass man nach dem neuen Verfahren auch bei wechselnden Versuchsbedingungen constante Werthe erhält, und zwar ziemlich genau die Hälfte des Gesammtschwefels. Die andere Hälfte des Schwefels ist nach Abspaltung der ersten Hälfte in einer solchen Form vorhanden, dass sie durch Säure als Schwefelwasserstoff abgespalten werden kann, und dass sie sich ausserdem leicht zu Schwefelsäure oxydirt; diese Oxydation wird auch bei Anwendung von Zink in alkalischer Lösung 
nicht völlig verhindert, sondern nur, wenn das Kochen bei Ausschluss von Sauerstoff stattfindet.

Ein mit Amylmerkaptan (von Kahlbaum bezogen) ausgeführter Versuch hatte ein negatives Ergebniss.

Amylmerkaptan ca. 1/4 gr. wurde 10 Stunden mit 1 gr. Zink und alkalischer Bleilösung in Leuchtgasatmosphäre gekocht. Hierbei bildete sich kein Schwefelblei, zugleich ein Beweis dafür, dass das Durchleiten von Leuchtgas unter den gegebenen Bedingungen keinen Fehler bedingt.

\section{Resultate.}

Aus diesen Beobachtungen geht hervor, dass die Zinkmethode mit hinreichender Genauigkeit eine quantitative Bestimmung des mit Alkali abspaltbaren Schwefels gestattet, und zwar wurde erhalten aus Thioessigsäure und Sulfoharnstoff der gesammte Schwefel, aus Thiosulfat, Cystin und Cystein die Hälfte des Gesammtschwefels.

Wir verdanken $\mathrm{Krüger}$ eine umfassende Zusammenstellung betreffend das Verhalten von Verbindungen, deren Schwefel mit Sauerstoff nicht in direkter Bindung steht, zu alkalischer Bleilösung. Diese auf qualitative Versuche begründete $\mathrm{Zu}$ sammenstellung führt $\mathrm{zu}$ folgenden Sätzen.

I. Während die $\equiv \mathrm{C}$-SH Verbindungen (Merkaptane) im Allgemeinen von wässerigen Alkalien nicht angegriffen werden, tritt eine Zersetzung unter Bildung von Schwefelmetall, ein, wenn an den Kohlenstoff direkt 0 (Thiosäuren) oder eine $\mathrm{NH}_{2}$ Gruppe gebunden ist (Cystein ist hier das einzige Beispiel.)

II. Die Verbindungen der Form $=\mathrm{C}=\mathrm{S}$ zersetzen sich, soweit bekannt, mit Alkalien unter Bildung von Schwefelmetall.

III. Diejenigen Verbindungen, in welchen der Schwefel zwei C-Atome verknüpft ( $\equiv \mathrm{C}-\mathrm{S}-\mathrm{C} \equiv$ ), sind zum Theil unangreirbar für wässerige Alkalien, zum Theil werden sie zersetzt, jedoch stets ohne Bildung von Schwefelmetall.

IV. Die Verbindungen der Form $\equiv \mathrm{C}-\mathrm{S}-\mathrm{S}-\mathrm{C} \equiv$ scheinen im Allgemeinen unter Bildung von $\mathrm{SH}_{2}$ zersetzt $\mathrm{zu}$ werden, falls jedoch der Kohlenstoff mit $\mathrm{O}$ verbunden ist, unangreifbar $\mathrm{zu}$ sein. 
Es wird also durchaus nicht aus allen Verbindungen, welche den Schwefel in "nicht oxydirter Form» besitzen, Schwefel beim Kochen mit Alkali als Schwefelmetall abgespalten.

Quantitative Versuche über die Abspaltung des Schwefels aus bekannten Verbindungen liegen nur in geringer Anzahl vor. Für den Sulfoharnstoff und den Allylsulfoharnstoff fand Suter, dass der Schwefel durch 2stündiges Kochen mit Alkali quantitativ abgespalten wird. ${ }^{1}$ ) Sodann liegen noch von $\mathrm{Bau}$ mann und Goldmann ${ }^{2}$ ) sowie von Suter quantitative Versuche über Cystin vor. Baumann und Goldmann konnten aus Cystin durch 9stündiges Kochen 68,4 \% , Suter durch $33^{1} / 2$ stündiges Kochen $83 \%$ des Gesammtschwefels als Schwefelblei erhalten. Diese Werthe widersprechen den von mir gefundenen, unterliegen aber, da die Menge des abgespaltenen Schwefels einfach durch Wägung des getrockneten Niederschlags bestimmt wurde (s. Anmerk. 1), ernsten Bedenken.

In zwei Versuchen, die Baumann und Goldmann mit Harn, dem sie eine gewogene Menge Cystin zugesetzt hatten, anstellten, erhielten sie nur $36 \%$ und $32, \mathbf{a} \%$ des Gesammtschwefels als Schwefel-. blei.

Bei diesen Versuchen wurde nicht einfach der Niederschlag gewogen, sondern der ausgewaschene Niederschlag wurde mit Salzsäure zerlegt und der entweichende Schwefelwasserstoff in einer Vorlage mit

1) Aus $0,2928 \mathrm{gr}$. Sulfoharnstoff erhielt er statt $0,1232 \mathrm{gr}$. $\mathrm{S}$ in Form von $\mathrm{PbS} 0,1232 \mathrm{gr}$; aus $0,45001 \mathrm{gr}$. Allylsulfoharnstoff $0,1256 \mathrm{gr}$. S statt $0,1242 \mathrm{gr}$. S.

Dieses günstige Ergebniss stimmt zwar mit den Resultaten der Zinkmethode überein, beruht aber doch wohl darauf, dass sich die Fehler. des Verfahrens gegenseitig compensirten. Der durch Oxydation beim Kochen bedingte Verlust (s. vorher S. 18) wurde ausgeglichen dadurch, dass Suter den entstandenen Niederschlag nach dem Trocknen als. Schwefelblei wog und in Rechnung zog. Dies ist aber unzulässig, da das Schwefelblei beim Trocknen an der Luft zum Theil in schwefelsaures Blei übergeht; ausserdem enthält ein solcher Niederschlag, namentlich bei complicirteren Verbindungen, sicher Verunreinigungen, die mit dem Schwefelgehalte nichts zu thun haben. Hiervon konnte ich mich auch bei Cystin überzeugen.

2) Zeitschrift f. physiolog. Chemie. Bd. XII, S. 257. 
Bleiacetatlösung aufgefangen und der hier entstehende Niederschlag nach dem Auswaschen und Trocknen gewogen. Inwiefern die hier gefundenen niedrigen Werthe von den durch die Anwendung von Harn gesetzten veränderten Verhältnissen oder von der abweichenden Methode herrühren, entzieht sich der Beurtheilung. 1 )

Die oben mitgetheilten Versuche sprechen dafür, dass die von mir ermittelten Bedingungen bei jenen Formen der Schwefelbindung, welche sich qualitativ durch Bildung von Schwefelblei erkennen lassen, die Abspaltung zu einer quantitativ bestimmbaren machen. Die bei Cystin und Cystein (welches beim Kochen mit Alkali rasch in Cystin übergeht) gefundene Ausnahme, wonach von dem bisher für abspaltbar gehaltenen Schwefel nur die Hälfte zu erhalten ist, kann dieses Urtheil nicht beeinträchtigen, da die derzeitige Vorstellung von der Bindungsweise des Schwefels im Cystin nicht über jedem Zweifel steht, denn die Aneinanderlagerung zweier Cysteine unter Verlust von zwei Wasserstoff ist noch in anderer als in der von Baumann angenommenen Weise denkbar.

\section{Schwefelbestimmungen in Eiweisskörpern.}

Untersucht wurden krystallisirtes Serumalbumin (Pferd), krystallisirtes Eieralbumin, krystallisirtes Oxyhämoglobin (Pferd), Globin (Pferd), Globulin (Pferd).

1) B. und G. haben (l. c.), um zu prüfen, ob die Austreibung des Schwefelwasserstoffs und die nachherige Wägung des in der Vorlage entstandenen Schwefelbleiniederschlages zu brauchbaren Resultaten führt, anscheinend nur einen Kontrollversuch mit Schwefelblei gemacht, in welchem sie statt $0,095 \mathrm{gr}$. Ausgangsmaterial 0,0941 gr. Niederschlag in der Vorlage bekamen. Aber schon Presch (Virchow's Archiv. Bd. 119. 1890. S. 148-167) wies nach, dass man bei diesem Verfahren unmöglich so ideal übereinstimmende Werthe erwarten hönne, da sich bei Destillation von Schwefelblei mit Salzsäure im Kühlrohr constant ein Schwefelbelag bildet, was unbedingt einen Verlust zur Folge haben muss; möglicher Weise sei dieser Fehler allerdings nur gering. Nach von mir ausgeführten Versuchen geht die Zerlegung des Schwefelbleies und die Austreibung des Schwefelwasserstoffes beim Kochen mit Salzsäure im Kohlensäurestrom nur sehr langsam vor sich. Die Resultate sprachen durchaus

für die Ansicht Presch's, dass das günstige Versuchsergebniss von Ba umann und Goldmann nicht als Regel anzusehen ist. 
Krystallisirtes Serumalbumin wurde zuerst dargestellt und beschrieben von Gürber, ${ }^{1}$ ) später von $\mathrm{Michel},{ }^{2}$ ) der dasselbe analysirte, und von G. Meyer. ${ }^{3}$ ) Verwandt wurde zu meinen Untersuchungen fünfmal unkrystallisirtes, durch Kochen coagulirtes Serumalbumin, welches zunächst mit Wasser bis zum Verschwinden der Schwefelsäure, dann mit Alkohol und Aether gewaschen und bis zur Gewichtsconstanz getrocknet war. ${ }^{*}$ ) Das so erhaltene Präparat wurde der Elementaranalyse unterworfen, welche folgende Werthe ergab:5) C 52,95\%, H 6.96\%, $\mathrm{S} 1,94 \%$, während nach Michel das coagulirte Serumalbumin folgende Zusammensetzung hat: C. 53,08\%, H 7,1\%, N 15,93\%, S $1,90 \%$.

Das $\mathrm{zu}$ der Untersuchung verwandte Eieralbumin war nach dem Hofmeister'schen Verfahren dargestellt und mehrfach umkrystallisirt, dann ebenfalls coagulirt und wie das Serumalbumin weiter behandelt. ${ }^{6}$ )

Das Hämoglobinpräparat (Pferd) war dreimal nach der

1) Sitzungsber. der Würzburger phys.-med. Gesellschaft, 1894 u. 1895,,. und Nachtrag zu der Arbeit von Michel, S. 139-144.

2) Verh. d. physik.-med. Gesellsch. zu Würzburg N.F., Bd. XXIX, S. $117-138$.

3) G. Meyer, Beiträge zur Kenntniss der Krystallisation des Serumeiweiss. Dissertation. Würzburg, 1896.

4) Das Auswaschen wurde auf einem Seidenfilter vorgenommen, um eine Verunreinigung mit Fasern zu vermeiden.

5) 1. C- und H-Bestimmung im offenen Rohr mit Kupferoxyd und vorgelegter reducirter Spirale.

Analyse I. 0,3199 gr. lieferten 0,1982 gr. $\mathrm{H}_{2} \mathrm{O}=6,88 \% \mathrm{H}$ und $0,6190 \mathrm{gr} . \mathrm{CO}_{2}=52,77 \% \mathrm{C}$.

Analyse II. $0,2663 \mathrm{gr}$. lieferten $0,1673 \mathrm{gr} . \mathrm{H}_{2} \mathrm{O}=6,98 \% \mathrm{H}$ und $0,5147 \mathrm{gr} . \mathrm{CO}_{2}=52,71 \% \mathrm{C}$.

2. Aschebestimmung $0,887 \mathrm{gr}$. lieferten $0,0035 \mathrm{gr}$. Asche $=0,4 \%$.

3. Gesammtschwefelbestimmung (v. Asboth) $0,4243 \mathrm{gr}$. lieferten 0,0604 gr. $\mathrm{Ba} \mathrm{SO}_{4}=1,93 \% \mathrm{~S}$.

6) Das Präparat verdanke ich Herrn H. Schneider. Die Analysen betreffs des Eieralbumins sind schon von Hofmeister (diese Zeitschr., Bd. XXIV, S. 168) mitgetheilt. 
Hoppe-Seyler'schen Methode umkrystallisirt, das Globinpräparat in anderweitig mitgetheilter Weise gewonnen. ${ }^{1}$ )

Das Serumglobulinpräparat (Pferd) war von dem Fibrinogen durch Zusatz von 3 Volumen concentrirter Ammonsulfatlösung aut 7 Volume durch Ammonoxalat ungerinnbar gemachtes Pferdeplasma befreit. ${ }^{2}$ ) Aus dem Filtrat wurde das Globulin durch Zusatz von soviel Ammonsulfat, dass eine halbgesättigte Lösung resultirte, ausgefällt. Dieser Globulinniederschlag wurde zum Reinigen mehrfach durch Einengen bei Zimmertemperatur aus verdünnter Ammonsulfatlösung ausgefällt. Hierbei schied sich das Globulin in schönen grossen Kugeln (Globuliten) aus, die aber selbst nach vielmaligem Ausfällen keine Uebergänge zu Krystallformen zeigten.

Die Gesammtschwefelbestimmungen wurden nach der von v. Asboth herrührenden Methode durch Schmelzen mit Natriumsuperoxyd und Soda vorgenommen. Diese Methode wurde als einerseits der einfacheren und weniger zeitraubenden Handhabung wegen, andererseits wegen der ausgezeichneten Genauigkeit zur Schwefelbestimmung im Eiweiss besonders geeignet, von Düring empfohlen. ${ }^{3}$ ) Durch zahlreiche Analysen habe ich mich überzeugen können, dass dieser Methode die ihr zugeschriebene Genauigkeit in der That zukommt. Die Bestimmung des abspaltbaren Schwefels geschah in der vorher beschriebenen Weise (s. Seite 20); das Kochen wurde in der Regel 10 Stunden und länger fortgesetzt.4) Für das Serumalbumin wurden auch zwei Versuche mit 10 stündigem Kochen in Leuchtgasatmosphäre gemacht, die dieselben Werthe ergaben, wie nach der Zinkmethode.

Die gefundenen Mittelwerthe sind aus der folgenden $\mathrm{Zu}$ sammenstellung ersichtlich.

1) Die näheren Angaben und Belege betreffs des Hämoglobin- und Globinpräparates siehe diese Zeitschr., Bd. XXIV, S. 449-481.

2) S. Reye, Ueber Nachweis und Bestimmung des Fibrinogens. Diss. Strassburg, 1898.

3) Zeitschr. f. physiol. Chemie, Bd. 22, 1896, S. 281-284.

4) Durch Kontrollversuche habe ich mich überzeugt, dass man durch längeres Kochen keine anderen Werthe erhält. 


\begin{tabular}{c|c|c|c|c|c}
\hline \hline & $\begin{array}{c}\text { Serum- } \\
\text { albumin }\end{array}$ & Eieralbumin & Hämoglobin & Globin & Globulin \\
\hline \begin{tabular}{c} 
a) Gesammtschwefel \\
\hline $\begin{array}{c}\text { Schwefel } \\
\text { b) abspaltbarer }\end{array}$
\end{tabular} & $1,89 \%$ & $1,18 \%$ & $0,43 \%$ & $0,42 \%$ & $1,38 \%$ \\
\hline a:b & $3: 2,03$ & $2: 0,83$ & $2: 0,88$ & $2: 0,95$ & $2: 0,91$ \\
\hline
\end{tabular}

Analytische Belege.

a) Gesammtschwefelbestimmungen.

I. Serumalbumin (s. Seite 28). Als Gesammtschwefel urde das Mittel aller bisher an krystallisirtem Serumalbumin ausgefühten Analysen gerechnet.

2. Eieralbumin. 0,582 gr. Eieralbumin lieferten 0,052 ๕. $\mathrm{Ba} \mathrm{SO}_{4}$ $=1,24 \% \mathrm{~S}, 0,593 \mathrm{gr}$. Eieralbumin lieferten 0,0547 gr. $\mathrm{Ba} \mathrm{SO}_{4}=, 27 \% \mathrm{~S}$.

Hofmeister fand (diese Zeitschrift, Bd. XVI, S. 187-19) 1,01\% und 1,18\%. Mittlerer Gesammtschwefelgehalt 1,18\%.

3. Den Gesammtschwefelgehalt des Hämoglobins und Gloins siehe diese Zeitschrift, Bd. XXIV, S. 464 u. 469.

4. Globulin. 0,3584 gr. Globulin lieferten $0,0356 \mathrm{gr}$. $\mathrm{Ba} \mathrm{SO}_{4}=1,38 \% \mathrm{~S}$,

$$
\begin{aligned}
& 0,1059 \gg \quad \text { » } 0,1051 \times \mathrm{Ba} \mathrm{SO}_{4}=1,41 \% \mathrm{~S} \text {. } \\
& 0,0934 \text { » } \gg 0,0886 \gg \mathrm{BaSO}_{4}=1,35 \% \mathrm{~S} \text {. }
\end{aligned}
$$

Mittlerer Gesammtschwefelgehalt 1,38\%.

b) Bestimmungen des abspaltbaren Schwefels.

$$
\text { A. Zinkmethode. }
$$

1. Serumalbumin.

I. 0,974 gr. Serumeiweiss, 11 Stunden gekocht.1) Gefunden 0,0923 gr. $\mathrm{Ba} \mathrm{SO}_{4}=1,30 \% \mathrm{~S}$.

II. $0,800 \mathrm{gr}$. Serumeiweiss, 11 Stunden gekocht. Gefunden $0,0774 \mathrm{gr} . \mathrm{Ba} \mathrm{SO}_{4}=1,32 \% \mathrm{~S}$.

III. 1,104 gr. Serumeiweiss, 11 Stunden gekocht. Gefunden 0,1016 gr. $\mathrm{Ba} \mathrm{SO}_{4}=1,26 \% \mathrm{~S}$.

B. Kochen in Leuchtgasatmosphäre.

I. 0,798 gr. Serumeiweiss, 10 Stunden gekocht. Gefunden 0,0707 gr. $\mathrm{Ba} \mathrm{SO}_{4}=1,22 \% \mathrm{~S}$.

II. 0,952 gr. Serumeiweiss, 15 Stunden gekocht. Gefunden 0,0903 gr. $\mathrm{Ba} \mathrm{SO}_{4}=1,30 \% \mathrm{~S}$.

1) Beim Kochen muss man Anfangs sehr vorsichtig seil da die Lösungen zu Beginn stark schäumen. 
2. Eieralbumin. Zinkmethode.

0,870 gr. Eieralbumin, 12 Sunden gekocht. Gefunden 0,0311 gr. $\mathrm{Ba} \mathrm{SO}_{4}=0,49 \%$.

Die zu dem Versuche verwandte Menge war, namentlich in Anbetracht des niedrigen Gesammtschwefelgehaltes, etwas gering, wodurch die Zuverlässigkeit der Analyse beeinträchtigt ist. Leider stand mir kein Material zu einem Kontrollversuche zur Verfügung.

3. Oxyhämoglobin. Zinkmethode.

I. 3,586 gr. Oxyhämoglobin, 11/2 Stunden gekocht. Gefunden 0,0471 gr. $\mathrm{Ba} \mathrm{SO}_{4}=0,18 \% \mathrm{~S}$.

II. 2,405 gr. Oxyhämoglobin, 11/2 Stunden gekocht. Gefunden 0,0364 gr. $\mathrm{Ba} \mathrm{SO}_{4}=0,20 \% \mathrm{~S}$.

Also im Mittel $0,19 \% \mathrm{~S}$.

4. Globin. 1,060 gr. Globin, 9 Stunden gekocht. Gefunden $0,0150 \mathrm{gr} . \mathrm{Ba} \mathrm{SO}_{4}=0,2 \% \mathrm{~S}$.

5. Globulin. Zinkmethode.

I. $1,123 \mathrm{gr}$. Globulin, 10 Stunden gekocht. Gefunden 0,0434 gr. $\mathrm{Ba} \mathrm{SO}_{4}=0,53 \% \mathrm{~S}$.

II. 1,526 gr. Globulin, 10 Stunden gekocht. Gefunden $0,074 \mathrm{gr} . \mathrm{Ba} \mathrm{So}_{4}=0,66 \% \mathrm{~S}$.

III. $1,184 \mathrm{gr}$. Globulin, 10 Stunden gekocht. Gefunden 0,0597 gr. $\mathrm{Ba} \mathrm{SO}_{4}=0,69 \% \mathrm{~S}$.

Also im Mittel 0,63\%.

Aus den angeführten Versuchen geht hervor, dass sich aus den untersuchten Eiweisskörpern durch Kochen mit Alkali unter Sauerstoffausschluss ein bestimmter und zwar erheblicher Antheil des Schwefels als Schwefelmetall abspalten lässt.

Für das krystallisirte Serumalbumin ergibt sich dabei ein Verhältniss von Gesammtschwefel zu abspaltbarem Schwefel wie genau 3:2. Darnach müssen im Serumalbumin drei Schwefelatome oder ein Vielfaches von drei vorhanden sein, und kommt ihm ein Molekulargewicht von 5100 oder einem Multiplum hiervon $\mathrm{zu}$.

Neuerdings macht Gürber ${ }^{1}$ ) folgende Angabe: «Im Serumalbumin ist das Mengenverhältniss des fest und locker gebundenen Schwefels $2: 3$, das Molekül enthält also mindestens 5 Schwefelatome. Diese Zahl ist zu verdoppeln, weil das Serumalbumin in wenigstens 7 . schwefelhaltige Verdauungsprodukte zerfällt, von denen 3 den Schwefel auch in beiden Formen enthalten.» Diese Angaben, offenbar auf eigenen Beobachtungen

1) Leitfaden der Physiologie des Menschen. Von F. Schenk und A. Gürber, 1897, S. 23. 
Gürb er's beruhend, lassen sich, solange eingehendere Mittheilungen über die angewandten Bestimmungsmethoden nicht vorliegen, schwer beurtheilen. Ich will nur erwähnen, dass, wenn im Serumalbumin das von Gürber angegebene Verhältniss bestände, 1,13\% Schwefel sich als Schwefelmetall abspalten lassen müssten, eine Zahl, die den von mir gefundenen Werthen zwar nahe steht, aber doch wohl ausserhalb der Fehlergrenze der von mir angewandten Methode liegt.1) Jedenfalls zeigt aber diese Ueberlegung, dass diese Schwefelbestimmungen allein für die Berechnung der Molekulargrösse nur einen beschränkten Werth besitzen, da eine fast in die Bestimmungsfehler fallende Differenz die Molekulargrösse um fast das Doppelte vergrössert, so dass es dringend erforderlich ist, die Schlussfolgerungen aus den Schwefelzahlen durch anderweitige Feststellungen zu sichern, wie es Gürber in der That durch Bestimmung der Anzahl der Verdauungsprodukte zu thun versucht hat.

Das für das Serumalbumin Gesagte gilt in entsprechender Weise auch für die anderen untersuchten Eiweisskörper. Hervorgehoben sei nur, dass dem Pferdehämoglobin dem Eisengehalte nach zwei Schwefelatome zukommen müssen, wie denn auch die oben gegebenen Zahlen diese Annahme unabweisbar machen. Da auch dem Globin selbst zwei Schwefelatome zukommen, ist es wahrscheinlich geworden, dass im Pferdehämoglobin je ein, 2 Schwefelatome enthaltendes Gilobinmolekül mit einem Hämatinmolekül verbunden ist. ${ }^{2}$ )

Ueber die nähere Art und Weise der Bindung des Schwefels ist im Vorliegenden nichts ausgesagt. Wie oben erwähnt, hat Krüger für eine grosse Anzahl von Verbindungen, deren Schwefel nicht in direkter Bindung mit Sauerstoff steht, das Verhalten gegen alkalische Bleilösung zusammengestellt, wobei sich ergab, dass viele dieser Verbindungen, mit Alkali gekocht, keinen Schwefel abspalten. Krüger wies daher die zuerst von Fleitmann aufgestellte Behauptung, dass im Eiweiss

1) Die von frïheren Untersuchern gefundenen Werthe habe ich hier nicht eingehender erwähnt, da dieselben mit den oben erwähnten Fehlern behaftet sind und sich daher mit meinen Zahlen kaum vergleichen lassen. Ich will nur hervorheben, dass meine Werthe ganz bedeutend höher liegen, so dass z. B. Krüger (l. c.) das umgekehrte Verhältniss für das Eiweiss annahm, indem er (bei Eieralbumin) nur 1/4 des Gesammtschwefels als Schwefelalkali abspalten konnte.

2) Vergl. Zeitschr. f. physiol. Chemie, Bd. XXIV, S. 472. 
ein Theil des Schwefels (der durch Alkali nicht abspaltbare) oxydirt, mit Sauerstoff direkt verbunden ist, zurück, indem er statt dessen mit Nasse von locker und fest gebundenem Schwefel spricht. Trotzdem findet man auch noch in den neuesten Lehrbüchern die Angabe, dass der durch Alkali nicht abspaltbare Schwefel in oxydirter Form vorhanden sei. ${ }^{1}$ )

Eine Klarstellung ist durch meine Versuche insofern gegeben, als das Vorhandensein zweier verschiedenartig gebundenen Schwefelformen im Eiweissmolekül durch eine in ihrer Wirkungsweise bekannte Methode bewiesen ist. Bis jetzt war es immerhin möglich, dass der gefundene Unterschied durch Fehler in der Methode vorgetäuscht wurde. In Betreff des nicht abspaltbaren Theils des Schwefels besteht nach wie vor zu Recht, dass man nicht von einem "oxydirten Schwefel » reden darf, sondern nur von einem «nicht durch Alkali abspaltbaren Schwefel.»

Nach einer kürzlich von Drechsel ${ }^{2}$ ) ausgesprochenen Vermuthung ist ein Theil des Schwefels im Eiweiss als vierwerthiger Schwefel, etwa in der Form der Thetinkörper enthalten. Da dieser Schwefel anscheinend als Alkylsulfid zur Abspaltung gelangt, würde derselbe beim Kochen mit Alkali nicht bleischwärzend wirken. Die Drechsel'sche Vermuthung widerspricht also unseren anderweitigen Erfahrungen keineswegs.

Es ist von Interesse, dass nach der Zinkmethode sich aus dem Hämoglobin und dem Globin Schwefel als Schwefelmetall abspalten lässt, da aus diesen Substanzen beim einfachen Kochen mit Alkali und Blei sich kein Schwefelblei bildet. ${ }^{3}$ ) Untersuchungen über den Verlauf der Abspaltung des Schwefels durch Kochen mit Alkali, wie sie zuerst von O. Nasse ${ }^{4}$ ) vorgenommen wurden, haben, was auch von allen Nach-

1) Neumeister, Lehrbuch der physiol. Chemie. 2. Aufl. S. 23. Gürber (u. Schenk) Leitfaden der Physiologie. S. 23.

2) Centralblatt f. Physiologie X, 1896, S. 529-530.

3) Malerba zählt (l. c.) das Globulin unter den Eiweisskörpern auf, welche, mit Alkali und Blei gekocht, kein Schwefelblei bilden. Mein Globulinpräparat wirkte, mit $30 \%$ iger Natronlauge gekocht, reichlich bleischwärzend.

4) 1. c. 
untersuchern bestätigt worden ist, ergeben, dass die Abspaltung zunächst ziemlich rasch, aber allmählich immer langsamer erfolgt, bis zu einer Grenze, wo überhaupt keine Schwefelabspaltung durch Bleischwärzung mehr nachweisbar ist. Diese Grenze ist je nach der Concentration des verwandten Alkalis verschieden. Jedenfalls geht aus diesen Untersuchungen hervor, dass mit sinkendem Schwefelgehalt die Abspaltung allmählich aufhört. Anderseits ist beobachtet worden, dass Eiweisskörper, die von vornherein einen niedrigen Schwefelgehalt haben, anscheinend bei der Bleireaction wenig oder gar keinen Schwefel abspalten. Danilevsky ${ }^{1}$ ) geht sogar so weit, aus dem Verhalten gegenüber alkalischer Bleilösung einen direkten Schluss auf den Gesammtschwefelgehalt des betreffenden Körpers für möglich zu halten. Danilevsky kommt hierbei zu einer auffallenden Uebereinstimmung zwischen dem Schwefelgehalt der Eiweisskörper, die überhaupt keinen abspaltbaren Schwefel zu enthalten scheinen, und der Menge des aus Körpern mit höherem Schwefelgehalt abspaltbaren Schwefels. Anscheinend kommt man in beiden Fällen an eine Grenze, wo die Abspaltung des Schwefels so langsam erfolgt, dass ohne besondere Vorsichtsmassregeln die Oxydation desselben mit der Abspaltung gleichen Schritt hält. Dies erklärt, warum nach der Zinkmethode in solchen Körpern noch wesentliche Mengen Schwefel als Sulfidschwefel nachweisbar sind.

Ein sehr lehrreiches Verhalten bietet auch in dieser Hinsicht das Thiosulfat dar. Kocht man Thiosulfat, welches, mit $30 \%$ iger Natronlauge an der Luft erhitzt, wie Seite 20 erwähnt, keinen Schwefel abspaltet, in Leuchtgasatmosphäre, so tritt bei Gegenwart von Blei ganz langsam ein allmählich zunehmender Schwefelbleiniederschlag auf, offenbar weil hier die Bedingungen für eine Oxydation nicht gegeben sind. Auch nach 15 stündigem Kochen ist die gebildete Menge Schwefelblei noch gering und entspricht keineswegs der verlangten Menge. Ist die verwendete Natronlauge höher concentrirt, so tritt auch an der Luft eine Bildung von Schwefelblei ein, da nunmehr die Abspaltung über die Oxydation überwiegt. Es besteht also für die Zinkmethode auch beim Thiosulfat, gegenüber dem einfachen Kochen mit Alkali, kein qualitativer Unterschied, sondern nur ein quantitativer.

Zum Schluss seien anhangsweise einige Versuche angeführt, welche lehren, dass das eingeschlagene Verfahren auch bei Untersuchung über die Bindungsweise des Schwefels im Harn von Nutzen sein dürfte.

Harn bildet unter normalen Verhältnissen beim Kochen mit alkalischer Bleilösung kein Schwefelblei. Nach der Zinkmethode erhält man jedoch wesentliche Mengen Schwefelmetall.

1) l. c. 
Menschenharn I. a. Gesammtschwefelsäurebestimmung. $100 \mathrm{ccm}$. lieferten 0,378 gr. $\mathrm{BaSO}_{4} \mp 0,052 \% \mathrm{~S}$.

b. Bestimmung des abspaltbaren Schwefels.

$100 \mathrm{ccm}$. lieferten (11 Stunden gekocht) $0,028 \mathrm{gr} . \mathrm{BaSO}_{4}=0,0038 \% \mathrm{~S}$, also $7,5 \%$ des oxydirten Schwefels.

Menschenharn II. a. Gesammtschwefelbestimmung (v. Asboth).

1) $10,0 \mathrm{ccm}$. lieferten $0,0521 \mathrm{gr} . \mathrm{BaSO}_{4}=0,071 \% \mathrm{~S}$

2) $10,0 \mathrm{ccm}$. lieferten $0,055 \mathrm{gr} . \mathrm{BaSO}_{4}=0,075 \% \mathrm{~S}$.

b. Gesammtschwefelsäurebestimmung.

$20 \mathrm{ccm}$. lieferten $0,075 \mathrm{gr} . \mathrm{BaSO}_{4}=0,051 \% \mathrm{~S}$.

c. Bestimmung des abspaltbaren Schwefels.

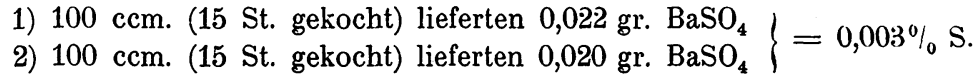

Also besteht annähernd dasselbe Verhältniss wie bei Harn I.

Es bietet sich somit die Möglichkeit, den reducirten Schwefel, wenigstens zum Theil, in dieser Weise direkt $\mathrm{zu}$ bestimmen, während man früher nur auf Differenzberechnungen angewiesen war.

Es werden jedoch auch hier noch weitere Untersuchungen lehren müssen, auf welche Art von Schwefelverbindungen die durch die Zinkmethode bestimmbare Sulfidbildung zurückzuführen ist.

Strassburg, im Februar 1897. 